\title{
Gradhiva
}

Julien Bonhomme, Les Voleurs de sexe. Anthropologie d'une rumeur africaine

Paris, Seuil, coll. « La librairie du XxI ${ }^{\mathrm{e}}$ siècle ", 2009, 192 p.

\section{Giordana Charuty}

\section{CpenEdition}

\section{Journals}

Édition électronique

URL : http://journals.openedition.org/gradhiva/1986

DOI : $10.4000 /$ gradhiva. 1986

ISSN : 1760-849X

Éditeur

Musée du quai Branly Jacques Chirac

Édition imprimée

Date de publication : 24 novembre 2010

Pagination : 237-238

ISBN : 978-2-35744-029-6

ISSN : 0764-8928

Référence électronique

Giordana Charuty, «Julien Bonhomme, Les Voleurs de sexe. Anthropologie d'une rumeur africaine », Gradhiva [En ligne], 12 | 2010, mis en ligne le 24 novembre 2010, consulté le 21 septembre 2020. URL: http://journals.openedition.org/gradhiva/1986 ; DOI : https://doi.org/10.4000/gradhiva.1986

Ce document a été généré automatiquement le 21 septembre 2020.

(c) musée du quai Branly 


\section{Julien Bonhomme, Les Voleurs de sexe. Anthropologie d'une rumeur africaine}

Paris, Seuil, coll. « La librairie du XxI ${ }^{\mathrm{e}}$ siècle », 2009, 192 p.

\section{Giordana Charuty}

\section{RÉFÉRENCE}

Julien Bonhomme, Les Voleurs de sexe. Anthropologie d'une rumeur africaine. Paris, Seuil, coll. « La librairie du XXI siècle », 2009, 192 p.

1 Ce livre est à la fois l'analyse d'un fait social de grande ampleur et un discours de la méthode quant aux problèmes que posent, à l'anthropologie, les transformations sociologiques qui affectent les objets classiques de la discipline. Lorsqu'une singulière accusation d'agression magique prend la dimension d'une rumeur transnationale qui se diffuse, depuis le début des années 1990, dans une vingtaine de pays de l'Afrique subsaharienne, en provoquant plusieurs morts pour chaque épisode recensé, comment le chercheur peut-il encore mettre en œuvre les exigences d'une description ethnographique?

2 C'est en prenant la mesure de l'épaisseur temporelle et de l'extension géographique d'un «fait divers » publié en mars 2001 par le quotidien gabonais L'Union que Julien Bonhomme a entrepris de répondre à cette question. L'article diagnostiquait l'emprise d'une psychose collective au sein de la deuxième grande ville du pays, Port-Gentil, déclenchée par cinq cas de "vol de sexe " où les présumés coupables de faire disparaître magiquement le pénis d'un inconnu croisé dans la rue étaient, à chaque fois, les victimes bien réelles des représailles, aussi violentes que soudaines, de la foule assemblée. Refusant ce langage de la pathologie sociale, immédiatement mobilisé par une écriture journalistique qui ne fait que prolonger les analyses sociologiques les plus classiques de la rumeur, l'auteur se propose de décrire dans sa positivité un 
fonctionnement «normal », « ordinaire » de la communication sociale. Pour ce faire, il reprend en partie le modèle épidémiologique des représentations élaboré par Dan Sperber pour l'articuler à une description pragmatique de chacune des variantes d'un "épisode", considéré comme un tout indissociable d'actions, d'émotions et de représentations.

Pour mener à bien cet "essai d'anthropologie pragmatique d'une rumeur» (p. 35), Julien Bonhomme commence par reconstituer les temps, les lieux, les rythmes de la propagation des accusations de « vol de sexe » en croisant les données fournies par de nombreux articles de presse - qui participent, de fait, à leur qualification et à leur propagation - et par les publications savantes - en sciences sociales, en psychiatrie, en psychologie sociale - qui se sont employées à combattre des croyances à ce point irrationnelles. Cartes à l'appui, il montre ainsi que, limitée jusqu'à la fin des années 1980 au Nigeria et à ses zones frontalières, la rumeur s'est diffusée en Afrique de l'Ouest et centrale à travers des cycles de réapparition périodique qui paraissent s'intensifier ces dernières années, sans que l'on puisse présumer de son extension à d'autres régions d'Afrique. D'autre part, n'hésitant pas à recourir à un élémentaire traitement statistique en appliquant un même questionnaire à tous les épisodes suffisamment documentés, l'auteur peut dégager l'existence d'un scénario qui se trouve reproduit, à l'identique, à travers les différents épisodes : des inconnus se croisent dans un lieu public ou se côtoient dans des transports collectifs ; un frôlement accidentel, un regard appuyé, des salutations inattendues, une demande de renseignements, une poignée de mains pour conclure une transaction commerciale produisent, chez la "victime ", la conviction de disparition ou de rétrécissement des organes génitaux; dans les cas les plus dramatiques, le « coupable » est bastonné, lapidé, brûlé vif par une foule anonyme.

4 Comparées à toutes les utilisations sorcellaires du corps et de ses fragments, l'expérience et l'accusation de vol de sexe apparaissent, alors, comme une variété singulière d'agression magique - c'est-à-dire d'interprétation d'une expérience émotionnelle de peur - qui menace, de manière quasi exclusive, la jeunesse masculine. Mais, rejetant les explications trop vagues en termes de crise de l'identité masculine, Julien Bonhomme s'appuie sur sa propre ethnographie au Gabon - des entretiens avec les témoins et les acteurs - pour identifier les formes d'interaction sociale susceptibles de donner naissance à cette interprétation. Plutôt que de s'en remettre au répertoire des explications fonctionnalistes qui ont pu être mobilisées pour rendre compte des affinités entre logiques de la rumeur et accusations sorcellaires, l'auteur emprunte à l'ethnographie de la sociabilité urbaine proposée par Georg Simmel et Erving Goffman les éléments permettant de caractériser, de manière positive, la singularité des interactions dans l'espace public urbain. Transposée à la ville africaine, cette perspective conduit à montrer, de manière convaincante, que ces accusations à première vue rocambolesques, mais aux conséquences parfois dramatiques, ne renvoient à rien d'autre qu'à la mise en échec des codes de conduite qui neutralisent la dangerosité potentielle d'interactions anonymes dans l'espace public urbain, en somme un « malaise dans l'interaction » (p. 65).

5 Encore faut-il décrire le processus de cette mise en échec, autrement dit l'articulation la «boucle causale " précise l'auteur - entre des représentations disponibles (où la diffusion médiatique joue un rôle essentiel), des réactions émotionnelles et physiologiques de frayeur, et des inférences cognitives qui conduisent à présupposer 
une même intentionnalité prédatrice à l'œuvre, non plus dans les relations d'interconnaissance entre parents qui caractérisent la sorcellerie familiale dans le contexte villageois propre à l'Afrique de l'Ouest, mais dans les interactions aléatoires et anonymes du trafic urbain. En effet, rassemblées méthodiquement en un tableau, les propriétés des unes inversent systématiquement celles des secondes (p. 89).

6 À l'autre pôle de l'analyse, il s'agit de rendre compte, plus classiquement, de l'assimilation de l'inconnu à l'étranger: autrement dit, d'identifier la logique xénophobe qui préside à l'intégration des catégorisations ethniques - les Haoussa, les Nigérians, les Ouest-Africains - pour faire de l'atteinte magique au potentiel vital d'un individu ou d'un groupe une composante essentielle des tensions interethniques et des violences intercommunautaires. Enfin, à partir d'un corpus de cas qui s'étend du Sénégal au Congo, se trouvent exposées les ambivalences de la presse qui, tour à tour, alimente la rumeur et s'efforce de la faire disparaitre, ainsi que les embarras de la police et de la justice confrontées à l'instabilité des places de "victime» et de «coupable » et aux paradoxes des accusations d'escroquerie.

7 L'enquête - et c'est là son principal mérite - progresse donc en défaisant, au fur et à mesure, bon nombre de lieux communs. La rumeur, en tant que mode de communication, ne se caractérise ni par la variabilité de son contenu ni par la fausseté de l'information transmise. L'irrationalité des accusations ne saurait conduire au diagnostic de pathologie sociale et l'on ne saurait, davantage, établir de corrélation causale entre rumeur et anomie urbaine ou crise sociale. Ajoutons que la notion de " légende urbaine " n'est guère plus opérante en domaine européen qu'en domaine africain lorsque les chercheurs négligent la comparaison contrastive avec les formes villageoises d'un domaine homologue d'expériences contre-intuitives. À l'inverse, bien des récits recueillis en contexte villageois européen - comme ceux illustrant le danger pour les enfants d'accepter de la nourriture offerte par une personne inconnue pourraient être lus selon le principe, proposé ici, d'une transformation des règles de la sociabilité.

8 Toutefois, au terme de la lecture, certaines interprétations, tout d'abord écartées comme trop génériques, ne mériteraient-elles pas d'être réexaminées? Si le danger d'interactions anonymes dans l'espace public urbain ne menace pas de la même façon les hommes et les femmes, quel est l'équivalent pour elles de ce «malaise dans l'interaction urbaine »? « On ne se situe pas dans un contexte de gestion ritualisée de la sorcellerie », observe Julien Bonhomme (p. 80) en essayant de décrire, et non de juger, le déchaînement de violence collective. De fait, en deçà des spécialisations régionales, cette étude très stimulante invite les anthropologues à interroger plus avant ce processus de mutation qui, à côté des grandes entreprises thérapeutico-religieuses, fait place à une sorcellerie hors médiation et hors rituel. 


\section{AUTEURS}

\section{GIORDANA CHARUTY}

giordana.charuty@laposte.net 